This item was submitted to Loughborough's Research Repository by the author.

Items in Figshare are protected by copyright, with all rights reserved, unless otherwise indicated.

\title{
Numerical investigation into the loading behaviour of filters operating in the diffusional and interception deposition regimes
}

PLEASE CITE THE PUBLISHED VERSION

http://dx.doi.org/10.1016/j.jaerosci.2012.06.008

PUBLISHER

(C) Elsevier

VERSION

AM (Accepted Manuscript)

LICENCE

CC BY-NC-ND 4.0

\section{REPOSITORY RECORD}

Dunnett, Sarah J., and Charles F. Clement. 2012. "Numerical Investigation into the Loading Behaviour of Filters Operating in the Diffusional and Interception Deposition Regimes”. figshare.

https://hdl.handle.net/2134/11179. 
This item was submitted to Loughborough's Institutional Repository (https://dspace.lboro.ac.uk/) by the author and is made available under the following Creative Commons Licence conditions.

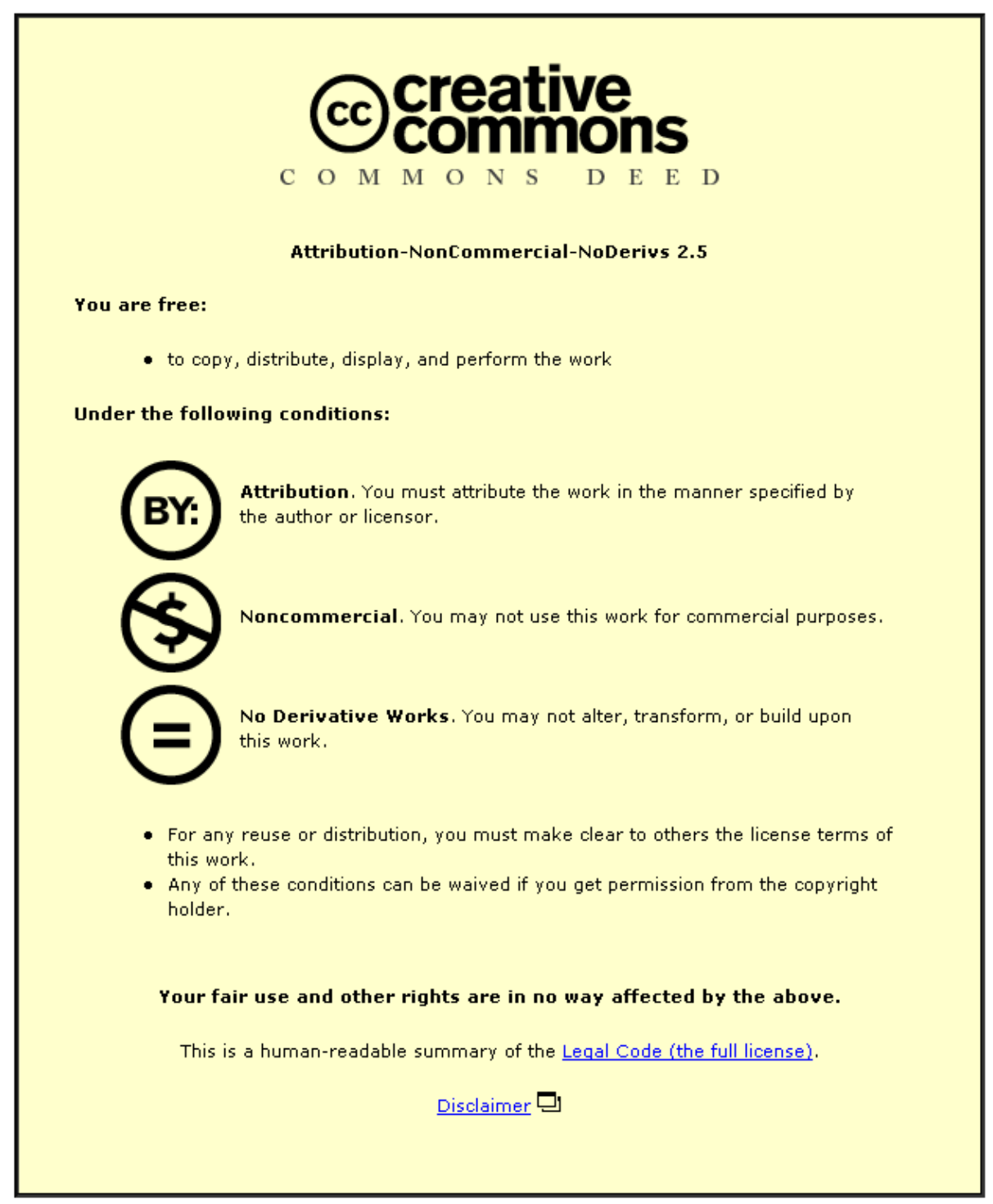

For the full text of this licence, please go to: http://creativecommons.org/licenses/by-nc-nd/2.5/ 
Numerical investigation into the loading behaviour of filters operating in the diffusional and interception deposition regimes.

\author{
Dr S.J. Dunnett ${ }^{1}$, Dr C.F. Clement ${ }^{2}$ \\ ${ }^{1}$ Department of Aeronautical and Automotive Engineering, Loughborough University, \\ Loughborough, Leics. LE11 3TU, UK \\ ${ }^{2} 15$ Witan Way, Wantage, Oxon, OX12 9EU, U.K.
}

\begin{abstract}
Using a previously developed theory, which allows for changes in gas flow from deposit growth, calculations are performed for deposition on an initially cylindrical fibre. The deposit is given a specified porosity through which the flow is calculated from Darcy's equation using the Beavers and Joseph (1967) boundary condition at the outer boundary. Results are obtained for different porosities $\varphi$, the volume fraction of space in the deposit, for flow conditions such that deposition occurs by the mechanisms of diffusion and interception, but not impaction (Stk $<1)$. Dependencies given in the literature of the deposit permeability on $\varphi$ are examined. The dominant mechanism is determined by the value of the parameter, $\mathrm{s}=\mathrm{R} / \delta$, where $\mathrm{R}$ is particle to fibre radius ratio and $\delta$ is the non-dimensional thickness of the flow diffusion layer. Where diffusion dominates, $\mathrm{s}<1$, increase in porosity does not significantly increase deposition, apart from the effect of its lower density. Where $s>1$ and interception dominates, deposition increases with increase in $\varphi$ and is significant for $\varphi \geq 0.9$ where more streamlines pass through the deposit. The shape of the deposit remains peaked at the front of the fibre, at the forward stagnation point, but, if a deposit has an initial flat front, it grows into a shape peaked away from the stagnation point, as observed by Kanaoka et al (1986). Possible reasons for this behaviour are discussed.
\end{abstract}

Keywords: Fibrous filter, numerical model, deposit, diffusion, interception. 


\section{Nomenclature}

C Cunningham correction factor

D Diffusion Coefficient

d Fibre width

$\mathrm{d}_{\mathrm{p}} \quad$ Particle diameter

F,G,H,M Functions of $\beta$ used in the numerical flow model.

g Function of $\beta$ describing the new surface of the fibre containing deposit.

k Permeability

$k^{\prime} \quad$ Non-dimensional value of $k\left(=\frac{4 k}{d^{2}}\right)$

$\mathrm{k}_{\mathrm{h}} \quad$ Hydrodynamic factor used in the flow model of Stechkina and Fuchs (1966)

$\mathrm{N} \quad$ Normal to the fibre surface

n Particle concentration

Pe Peclet number

Re Reynolds number of the flow

$\mathrm{R} \quad$ Ratio of particle and fibre radii

r Polar coordinate

$\mathrm{r}_{\mathrm{p}} \quad$ Particle radius

$\mathrm{S} \quad$ Deposit mechanism parameter $\left(=\frac{R}{\delta}\right)$

Stk Stokes number

$\mathrm{U}_{0} \quad$ Freestream velocity

U Fluid velocity

$\mathrm{U}_{\mathrm{r}} \quad$ Fluid velocity component in the r direction

$\mathrm{U}_{\theta} \quad$ Fluid velocity component in the $\theta$ direction

$\mathrm{x}, \mathrm{y} \quad$ Cartesian components

$\alpha \quad$ Packing fraction

$\alpha_{\mathrm{BJ}} \quad$ Coefficient in Beavers and Josephs (1967) boundary condition

$\beta \quad$ Angle from incident direction

$\delta \quad$ Non-dimensional thickness of the diffusion layer

$\varphi \quad$ Volume fraction of space in the deposit

$\lambda$ Constant related to the rate at which particles deposit upon the fibre surface 
$\eta \quad$ Particle collection efficiency

$\mu \quad$ Air viscosity

$\theta \quad$ Polar coordinate

$\rho \quad$ Density of air

$\rho_{p} \quad$ Density of particle

$\omega \quad$ Fluid vorticity

$\psi \quad$ Stream function 


\section{INTRODUCTION.}

The separation of particles from a flow by the use of fibrous filters is common practice. Examples are in the protection of individuals from harmful airborne particulate by the use of face masks, in air pollution control and many other applications. Such filters generally consist of numerous fibres, of varying sizes, which are positioned more or less normal to the flow. The flow passes through the regions between the fibres and particles are removed by attaching to the fibre surface. The mechanisms by which the particles are removed depend on various particle and flow properties, such as flow velocity, particle size, etc. As the deposit accumulates inside the filter the flow through it, and hence the efficiency with which further particles is collected, is altered.

In order to fully understand the behaviour of fibrous filters it is necessary to obtain a detailed description of the flow in the filter. As the structure of filters is very complex with the random orientation of the fibrous filters and the interference of the neighbouring fibres this is a difficult task and hence simplifications have been made. One approach which is widely adopted is the cell model initially developed independently by Kuwabara (1959) and Happel (1959). This approach although primarily considering a single fibre, takes into account the effects of the neighbouring fibres and is described in more detail in the following section. Many different numerical approaches have been adopted to solve the flow equations including the Boundary Element Method (BEM), see for example Hildyard et al (1985) and Konstandopoulos (2000), the control volume method, see for example Li and Marshall (2007) and Qian et al (2009) and the lattice-Boltzmann approach, Filippova and Hanel (1997) and Przekop et al (2003). Once the flow field is determined the movement of particles within the filter and their possible removal by the fibres can be investigated. Over the years, due to its importance, much work has been performed in this area. A lot of this work has considered the case of a clean filter, much of which is reviewed in Brown (1993), and this situation is now well understood. The feedback effects of the deposit formed and its dependence upon filter parameters are however less well understood and this is the area considered in this paper. As particles collect on the fibre surface, due to their finite size they also become part of the filter structure and hence affect its efficiency. Numerical simulations of such deposit are limited due to their considerable use of computing time and resources, see Jung and Tien (1993), Biggs et al (2003) , Karadimos and Ocone (2003) and Przekop et al (2003). 
As deposit builds up on the fibres, dendrites, branch like structures made up of deposited particles, are formed. Models of such structures have been developed by Payatakes and colleagues, e.g. Payatakes et al (1977), Payatakes and Gradon (1980). These models are however limited to the early stages of deposition due to the assumptions made in neglecting the effects of the structures formed upon the flow field.

The area on the fibres at which deposition takes place, and the shape of the deposit formed, will depend upon the dominant particle capture mechanism. A diagram outlining the relationship between the operating parameters and the deposit formed was given in Kanaoka et al (1986) and reproduced in Kasper et al (2010), see figure 1. In this figure Pe is the Peclet number, which is a measure of the relative magnitude of the diffusional motion of the particles and the convective motion of the air past the fibre and is given by:

$$
\mathrm{Pe}=\frac{\mathrm{U}_{0} \mathrm{~d}}{\mathrm{D}}
$$

where $\mathrm{D}$ is the coefficient of diffusion of the particles, $d$ the fibre diameter and $U_{0}$ the freestream velocity. $R$ is the interception parameter, $R=d_{p} / d$ where $d_{p}$ is the particle diameter and Stk is the Stokes number, which is the ratio of the particle stop distance to fibre diameter.

As can be seen in figure 1, when diffusional deposition is the dominant mechanism, small Pe and R, the particles are evenly distributed around the fibre with a relatively open pore structure. As the interception parameter increases for the same flow parameters Pe and Stk, the basic distribution of the deposit remains the same but the structure is more open. For large Stk, when inertial impaction is dominant the deposition is purely on the front of the fibre with the majority close to the stagnation point.

Recently a detailed study has been undertaken into the deposit onto, and subsequent performance of a single fibre, by Kasper and colleagues, Lehmann and Kasper (2002), Kasper et al (2009) and Kasper et al (2010). The studies were undertaken for situations where deposition is dominated by inertial impaction and interception (to the right on figure 1). The CFD simulations by Lehmann and Kasper (2002) demonstrated the importance of particle rebound upon deposit structure in the inertial regime and also found that simulations need to be three dimensional to accurately predict the deposit packing fraction. The experimental process undertaken by Kasper et al (2009) 
and Kasper et al (2010) generated much useful structural information about the deposit for particles where inertia, interception and bounce are important. It was found that the transition from compact deposits to the more dendritic structures as shown in the figure from Kanaoka et al, Figure 1 as R increases, is driven not by interception but by particle bounce.

The aims of this present study are to consider the loading behaviour of filters operating in the diffusional, and interception, deposition regimes (to the left and centre of Figure 1). A numerical model for loaded fibres developed in previous papers Dunnett and Clement (2006, 2009), subsequently referred to as I and II in this paper, is applied to consider the effects of deposit upon performance for various operating parameters. In the paper initially a brief description is given of cell models in general and the particular model of Dunnett and Clement outlined. This model assumes the deposit to form a smooth porous layer on the fibre surface, (similar to the shapes shown at the top of figure 1) the shape of this layer is dependent upon the deposition mechanism. Hence the model clearly only applies when R (referred to as $\kappa$ in I and II) $<<1$, the complex structure of the dendrites is not modelled and therefore the results obtained give a qualitative description of the effect of deposition. This model is then applied to consider the effects of the porous deposit upon the flowfield, and hence filter performance, for situations when diffusion, and then interception, are the main mechanisms of deposition. The deposition patterns are compared with those described previously by Kanaoka et al and hence the range of validity of the numerical model investigated.

In order to describe the growing deposit, we need to calculate the gas flow and then particle motion and resulting deposition in the flow. Starting with a clean fibre this leads to an initial deposit layer, and the whole process is then iterated but with a new surface for deposition and a possibly porous layer through which gas can flow. Formulation for the flow calculations, summarising work performed in I and II, is described in section 2, first for a clean fibre, then with the addition of a solid deposit, and finally in 2.3 including flow through a porous layer. In particular, the complex formulas derived in II required to implement the Beavers and Joseph boundary condition on the changing layer surface are included in 2.3. Particle motion and deposition from the flow by the two mechanisms of diffusion and interception is described in section 3, giving in 3.1 the key parameter determining which mechanism 
dominates. In section 3.2 we study various relations given in the literature between the gas permeability and the porosity of the deposit. In section 4, we describe the results of iterated deposition calculations, first for the diffusive regime in 4.1 and then for the interception regime in 4.2. Conclusions from the results in relation to the importance of deposit porosity and to the experiments of Kanaoka et al (1986) are given in section 5 .

\section{FLUID FLOW FORMULATION}

As mentioned in the previous section many of the earlier flow models have been based on the cell method. As the current model developed here is also based on this approach a description of the method is given below

\subsection{CELL MODEL}

In these models the assumption is made that the Reynolds number of the flow, Re, is small and hence creeping flow is assumed, where $\operatorname{Re}=\frac{\mathrm{d} \rho \mathrm{U}_{0}}{\mu} \rho$ is the density of the fluid and $\mu$ the fluid viscosity. The equations of motion for the flow are then:

$$
\begin{aligned}
& \nabla \mathrm{p}=\mu \nabla^{2} \underline{\mathrm{U}} \\
& \nabla \cdot \underline{\mathrm{U}}=0
\end{aligned}
$$

In these equations $\mathrm{p}$ is the fluid pressure. Assuming two dimensional flow, in terms of the streamfunction, $\psi$, equations (2) are written as:

$$
\nabla^{4} \psi=0
$$

which can be written in coupled form as:

$$
\begin{aligned}
& \nabla^{2} \psi=\omega \\
& \nabla^{2} \omega=0
\end{aligned}
$$

where $\omega$ is the fluid vorticity which is a measure of the tendency of the fluid to rotate or to cause rotation of suspended bodies during flow.

Equations (4) are then solved in the region around a single fibre, with the flow interference effect of the neighbouring fibres taken into account by the application of boundary conditions on the outer boundary of the region. This outer boundary is defined by a surface concentric with the fibre and at such a distance from the fibre 
that the packing fraction of the fibre within this surface is identical to that of the fibres within the filter, $\alpha$. This results in the diameter of the outer boundary, a, being defined by a $=\frac{\mathrm{d}}{\sqrt{\alpha}}$.

As the flow is determined in a limited region, assumed to be representative of the filter, it is known as a cell model. In the model of Kuwabara the boundary conditions applied were the no slip condition on the fibre surface and $\mathrm{u}=\mathrm{U}_{0} \cos \theta$ and zero vorticity on the cell boundary. The model of Happel only differed in that the shear stress was assumed zero on the cell boundary and not the vorticity.

In this present study cell models have also been adopted. In previous papers I and II, numerical models of the flow field and particle motion for the cases of a clean fibre and a fibre containing some deposit have been developed and validated. The model to determine the flow characteristics used the Boundary Element Method (BEM) and a brief summary of the main points is given here. More detail can be found in the original papers.

The fibres are modelled as infinitely long cylinders with their axis perpendicular to the flow. Hence the flow is two dimensional and the motion takes place in the plane perpendicular to the cylinder axis. A single fibre is considered and a cell model adopted. The Reynolds number of the flow is assumed to be small so that equations (2)-(4) are applicable. Before making any calculations all quantities were nondimensionalised, distances with respect to the fibre radius $d / 2$ and velocities with respect to the mean flow velocity $U_{0}$. The form of equation (4) is unchanged.

\subsection{CLEAN FIBRE + FIBRE CONTAINING A SOLID DEPOSIT.}

In the case of a clean fibre the solution domain is shown in Figure 2a where symmetry is taken into account. Equations (4) are solved, subject to the following boundary conditions:

(a) $\psi=0, \omega=0$ on $\mathrm{AB}$ and $\mathrm{CD}$

(b) $\psi=0, \psi^{\prime}=0$ on BC

(c) $\psi=\mathrm{h}_{1} \sin \theta, \omega=0$ on DA

In condition (b) prime denotes differentiation with respect to the outward normal to the surface BC and in condition (c) $h_{1}$ is the non-dimensional radius of the cell and is 
given by $\mathrm{h}_{1}=1 / \sqrt{\alpha}$ and $\theta$ is the standard polar coordinate. Details of the method of solution for the clean fibre are given in I.

Initially, for ease of calculation, the surface formed by depositing particles was assumed to be solid and hence the same method as for a clean fibre could be used to determine the flowfield for a fibre with deposit. The only difference being the shape of the boundary BC in figure 2a. This is also described in more detail in I. In order to more realistically model the deposit this was later extended and the deposited particles assumed to form a porous layer on the fibre.

\subsection{FIBRE CONTAINING POROUS DEPOSIT.}

Full details of the model developed for this case are given in paper II but the main points will be summarised here. The domain considered was that shown in Figure 2b, once again use was made of symmetry and AF is the symmetry boundary. The boundary of the porous layer formed by the deposited material, BE, is described, in non-dimensional terms, by the equation $r=1+\lambda g(\beta)$ where $g(\beta)$ is a numerical function of the angle $\beta=\pi-\theta$ that $r$ makes with the negative $x$ axis and $\lambda$ is the non-dimensional thickness of the deposit at the front of the fibre.

There are two regions to the flow field, the region outside the deposit where the flow satisfies equations (2) as in section (2.2), this is labelled region I in Figure 2b. The other region in which the flow is determined is inside the porous layer, denoted region II in Figure 2b. Also shown in the figure is a limiting particle trajectory for a particle of diameter $d_{p}$ which just touches the deposition surface. In the model described in II the flow inside the porous layer is assumed to be modelled by Darcy's law;

$$
\nabla \mathrm{p}=-\frac{\mu}{\mathrm{k}} \underline{\mathrm{U}}
$$

where $\mathrm{k}$ is the permeability of the porous media. This permeability is independent of the nature of the fluid but it does depend on the geometry of the porous medium. In the model developed the expression for $\mathrm{k}$ obtained from the Carman-Kozeny formula, equation (7), was adopted.

$$
k=\frac{\varphi^{3} d_{p}^{2}}{180(1-\varphi)^{2}}
$$


Where $\varphi$ is the porosity, which is the fraction of the porous media that is occupied by void space. Subscripts $\mathrm{F}$ and $\mathrm{P}$ were used to denote the flow variables in the regions I and II respectively. In region I $\psi_{F}$ satisfies equations (4) and in region II equation (6) can be written as;

$\nabla^{2} \psi_{\mathrm{p}}=0$

Equations (4) and (8) were solved, using the BEM, for the streamfunction in the two regions subject to the following boundary conditions;

(a) $\quad \psi_{\mathrm{F}}=0, \omega_{\mathrm{F}}=0$ on $\mathrm{AB}$ and $\mathrm{EF}$

(b) $\psi_{\mathrm{F}}=\mathrm{h}_{1} \sin \theta, \omega_{\mathrm{F}}=0$ on $\mathrm{AF}$

(c) $\quad \psi_{\mathrm{P}}=0$ on $\mathrm{BC}, \mathrm{CD}$ and $\mathrm{DE}$

and on the common boundary BE: $\frac{\partial \mathrm{p}}{\partial \mathrm{T}}$ and the normal velocity $\mathrm{U}_{\mathrm{N}}$ are continuous and the generalised Beavers and Joseph (1967) condition:

$\left(\mathrm{U}_{\mathrm{T}}^{\prime}+\frac{\partial \mathrm{U}_{\mathrm{N}}}{\partial \mathrm{T}}\right)_{\mathrm{F}}=\frac{\alpha_{\mathrm{BJ}}}{\sqrt{\mathrm{k}}}\left(\left(\mathrm{U}_{\mathrm{T}}\right)_{\mathrm{F}}-\left(\mathrm{U}_{\mathrm{T}}\right)_{\mathrm{P}}\right)$

is satisfied, where $T$ is the tangent to the boundary $B E$ and $\alpha_{\mathrm{BJ}}$ is a dimensionless slip parameter which depends on the material parameters that characterize the structure of the permeable material. This results in the following conditions on BE, see paper II for more detail:

(d) $\left.\quad \frac{\partial \psi}{\partial \mathrm{T}}\right|_{\mathrm{F}}=\left.\frac{\partial \psi}{\partial \mathrm{T}}\right|_{\mathrm{P}}$

(e) $\quad \frac{\psi_{\mathrm{P}}^{\prime}}{\mathrm{k}}=\omega_{\mathrm{F}}^{\prime}$

$$
\psi_{\mathrm{P}}^{\prime}=-\psi_{\mathrm{F}}^{\prime}+\frac{\sqrt{\mathrm{k}}}{\alpha_{\mathrm{BJ}}}\left[-\frac{\mathrm{F}(\beta)}{|\mathrm{M}(\beta)|^{2}} \omega_{\mathrm{F}}+2 \frac{\partial^{2} \psi_{\mathrm{F}}}{\partial \mathrm{T}^{2}}+\frac{\mathrm{G}(\beta)}{\mathrm{rF}(\beta)|\mathrm{M}(\beta)|} \psi_{\mathrm{F}}^{\prime}+\frac{\mathrm{H}(\beta)}{\mathrm{rF}(\beta)|\mathrm{M}(\beta)|} \frac{\partial \psi_{\mathrm{F}}}{\partial \mathrm{T}}\right]
$$

In condition (f) the functions $F(\beta), G(\beta), H(\beta)$ and $M(\beta)$ are given by: 


$$
\begin{aligned}
& F(\beta)=\lambda^{2}\left(\frac{d g}{d \beta}\right)^{2}+(1+\lambda g)^{2} \\
& G(\beta)=\lambda^{3}\left(\frac{d g}{d \beta}\right)^{2} \frac{d^{2} g}{d \beta^{2}}+3 \lambda^{2}\left(\frac{d g}{d \beta}\right)^{2}(1+\lambda g)+(1+\lambda g)^{3}-\lambda\left(\frac{d^{2} g}{d \beta^{2}}\right)(1+\lambda g)^{2} \\
& H(\beta)=2 \lambda^{2}\left(\frac{d g}{d \beta}\right)\left(\left(\frac{d^{2} g}{d \beta^{2}}\right)(1+\lambda g)-\lambda\left(\frac{d g}{d \beta}\right)^{2}\right) \\
& M(\beta)=\left(1+\lambda^{2}\left(g^{2}+\left(\frac{d g}{d \beta}\right)^{2}\right)+2 \lambda g\right)^{1 / 2}
\end{aligned}
$$

Once the streamfunction has been determined for either a clean fibre or one containing deposit, the velocities of the flow can be determined using:

$$
\mathrm{u}_{\mathrm{r}}=\frac{1}{\mathrm{r}} \frac{\partial \psi}{\partial \theta} \quad \text { and } \mathrm{u}_{\theta}=-\frac{\partial \psi}{\partial \mathrm{r}}
$$

\section{PARTICLE MODELLING}

\subsection{PARTICLE DEPOSITION.}

The basic quantities which determine whether incident aerosol particles are deposited on a fibre are their incident velocity, $\mathrm{U}_{0}$, diameter, $\mathrm{d}_{\mathrm{p}}$, of the assumed spherical particle, the nature of the gas (density, $\rho$, temperature, $T$, and viscosity, $\mu$ ) and characteristics of the fibres. These characteristics are initially specified by the fibre diameter, $\mathrm{d}$, and the geometric arrangement of the fibres which includes their packing fraction, $\alpha$, which we take to be uniform. We also assume that, following deposition, the porosity of the deposit is also uniform and is specified by a single value for the volume fraction of space, $\varphi$, in the deposit. The viscous gas flow through the deposit depends on both $\varphi$ and the particle diameter, a dependence we examine later. Neither uniformity assumption is entirely justified, and initial structure variability has been investigated experimentally (Lehmann et al 2003) and its effects by Schweers and Löffler (1994).

Furthermore, the porosity can depend on the deposition mechanism, which can vary with angle round a fibre, and also depth in the deposit ( Rodríguez-Pérez et al 2005). 
In the numerical model developed in I and II once the flow characteristics were determined, the motion of the particles in the flow and their deposition onto the fibre was considered. In filtration, neglecting electrical effects, the main mechanisms by which particles are deposited on the fibres are:

i) diffusional deposition, when the combined action of the fluid flow and the Brownian motion of the particle brings it into contact with a fibre.

ii) direct interception, when a particle following a streamline of the flow comes into contact with a fibre.

iii) inertial impaction, when a particle deviates from a streamline because of its own inertia and hence come into contact with a fibre.

iv) gravitational settling, when particles settle out of the air moving through the filter due to the influence of gravity.

The dominant mechanism that influences the deposition is dependent upon the size of the particles. In the earlier work presented in I and II small particles were considered where the main mechanism of deposition is diffusional deposition. In this case the equation to be solved for the non-dimensional particle concentration, $\mathrm{n}$, is:

$\mathrm{u}_{\mathrm{r}} \frac{\partial \mathrm{n}}{\partial \mathrm{r}}+\frac{\mathrm{u}_{\theta}}{\mathrm{r}} \frac{\partial \mathrm{n}}{\partial \theta}=\frac{2}{\operatorname{Pe}}\left(\frac{\partial^{2} \mathrm{n}}{\partial \mathrm{r}^{2}}+\frac{1}{\mathrm{r}} \frac{\partial \mathrm{n}}{\partial \mathrm{r}}\right)$

where particle concentration has been non-dimensionalised with respect to the particle concentration in the undisturbed flow.

Details of the method adopted to solve equation (14) and the boundary conditions imposed are given in I. The boundary conditions account for the effects of particle interception with the surface which introduces the dimensionless particle diameter $\mathrm{R}$. Intermediate size particles have negligible inertia, gravitational settling or Brownian motion and hence follow the flow streamlines. In this case the dominant deposition mechanism is direct interception and hence the limiting streamlines, those that come within a particle radius of the surface, need to be determined. This mechanism will be considered in this work.

Once the particle motion is modelled, the rate at which the particles deposit can be determined and hence the numerical function $g(\beta)$, which defines the surface formed by the deposit, determined. 
In I we found that the analytic behaviour of the solution of the transport equation (14) changes across the line where the value of the ratio of $\delta$ to $\mathrm{R}$ is 1 . Where the quantity, $\delta$, represents the thickness of the diffusion layer round the fibre, given by:

$\delta=\left(4 \mathrm{k}_{\mathrm{h}} / \mathrm{Pe}\right)^{1 / 3}$,

where the hydrodynamic factor, $\mathrm{k}_{\mathrm{h}}$, for an isolated cylinder is given by

$\mathrm{k}_{\mathrm{h}}=2-\ln \mathrm{Re}$,

and for a system of parallel cylinders by

$k_{h}=-1 / 2 \ln \alpha-3 / 4+\alpha-1 / 4 \alpha^{2}$.

Previously, Stechkina and Fuchs (1966) characterised this ratio by a parameter $\mathrm{S}=\mathrm{R} / \delta$.

and found that diffusion dominates deposition for $\mathrm{s}<1$, and interception dominates deposition for $\mathrm{s}>1$.

Considering the values of $\mathrm{s}$ for various physical conditions Figure 3 shows $\mathrm{s}$ as a function of particle size for various fibre sizes and freestream velocities. In the figure the packing fraction, $\alpha$, is taken to be 0.05 unless otherwise stated. Also shown on the figure is the line $s=1$. Hence the conditions for which the curves lie below the $s=1$ line correspond to situations when deposition is dominated by diffusion and those above by interception.

We find here that the value of $\mathrm{s}$ is also the most important parameter in determining whether or not porosity in the deposit subsequently increases the deposition rate. In the work presented here the numerical models developed earlier, and described briefly here, will be used to investigate the different deposition mechanisms of diffusional deposition and interception. The range of conditions and parameters which determine which of these mechanisms is dominant will be discussed. The shape of the deposit formed on the fibre for the different deposition mechanisms is investigated and the validity of assumptions made in the numerical models investigated.

\subsection{PERMEABILITY.}

The flow through the porous layer formed by the deposited particles is characterised by the permeability of the layer, $\mathrm{k}$, see equation (6). This permeability depends on the porosity and the structure of the deposit. In the model developed in I and II the permeability of the deposited material was given by the Carman-Kozeny formula, 
equation (7). This expression does not exhibit the correct limiting behaviour as $\varphi \rightarrow 1$, when the structure is very porous, see Vanni (2000) and Vainshtein et al (2004). In this case the permeability can be written as:

$$
k=\frac{f(\varphi)}{\varsigma(\varphi)} \frac{d_{p}^{2}}{4}
$$

where $f(\varphi)$ has the limiting behaviour,

$$
\mathrm{f}(\varphi) \rightarrow \frac{2}{9(1-\varphi)} \quad \text { as } \varphi \rightarrow 1
$$

which corresponds to the physical situation where each of the particles experiences drag as an isolated sphere, and $\zeta(\varphi)$ is a shielding coefficient to account for the fact that particles within the deposit will be in contact. Following the work of Vanni (2000) the shielding coefficient has been taken to be an exponential function given by:

$$
\zeta(\varphi)=1-0.6 \mathrm{e}^{-10(1-\varphi)},
$$

and the permeability models of Happel (1958):

$$
f(\varphi)=\frac{2}{9(1-\varphi)} \frac{\left(3-4.5 \gamma+4.5 \gamma^{5}-3 \gamma^{6}\right)}{\left(3+2 \gamma^{5}\right)}
$$

and Kim and Russel (1985)

$$
f(\varphi)=\frac{2}{9(1-\varphi)}\left(1+\frac{3}{\sqrt{2}} \gamma^{3 / 2}+\frac{405}{64} \gamma^{3} \ln \gamma+16.456 \gamma^{3}\right)
$$

have been considered, where $\gamma=(1-\varphi)^{1 / 3}$

The different values for k obtained by the different models is shown in Figure 4 where $4 \mathrm{k} / \mathrm{d}_{\mathrm{p}}{ }^{2}$ is shown as a function of $\varphi$ using the Carman-Kozeny model, equation (7), the Happel model, equations (19), (21) and (22) and the Kim and Russel model, equations (19), (21) and (23). The range of $\varphi$ shown is $0.6 \leq \varphi \leq 0.99$. As can be seen, for the larger values of $\varphi$ the models of Happel and Kim and Russel agree well, and they 
exhibit the correct limiting behaviour as $\varphi \rightarrow 1$, however they do differ significantly from the model of Carman-Kozeny. For the smaller values of $\varphi$ the model of Happel agrees well with the Carman-Kozeny model.

An example of the effect upon the flow field of the different models for $\mathrm{k}$ is shown in Figure 5 where the streamlines of the flow are shown near a fibre which has a porous layer on it for 2 different situations. In the figures, due to symmetry, only half of the fibre is shown and the porous region is enclosed within the dotted curve. Only the flow in the region close to the fibre + deposit is shown. In both figures shown, the porosity of the deposit is high, $\varphi=0.95$, and hence the Carman-Kozeny model is unlikely to be accurate.

In figure $5 \mathrm{a} d=40 \mu \mathrm{m}, \mathrm{U}_{0}=0.1 \mathrm{~m} / \mathrm{s}$ and $\mathrm{d}_{\mathrm{p}}=1 \mu \mathrm{m}$ which results in $\mathrm{s}=0.875$ and hence particles would be predominantly deposited by the mechanism of diffusion. The streamlines for the two models of Happel, and Kim and Russel were indistinguishable and hence only those for the Kim and Russel model are shown in the figure. As can be seen there is a small difference between this model and the Carman-Kozeny model. In figure $5 \mathrm{~b} d=20 \mu \mathrm{m}, \mathrm{U}_{0}=0.5 \mathrm{~m} / \mathrm{s}$ and $\mathrm{d}_{\mathrm{p}}=1 \mu \mathrm{m}$ which results in $\mathrm{s}=2.374$ and hence particles would be predominantly deposited by the mechanism of interception. In this case there is a small difference in the streamlines obtained using the Happel model and those obtained using the Kim and Russel model for the flow that travels through the deposit. However the difference is small. The results obtained using the CarmanKozeny model in this case, are significantly different from those using the other models.

As the models of Happel and Kim and Russel for permeability give similar flow patterns for the larger porosities and differ from those obtained using the CarmanKozeny model, the model of Happel will be adopted for the larger porosities in this work. This has a slightly simpler form than that of Kim and Russel, see equations (22) and (23), and has the correct limiting behaviour as $\varphi \rightarrow 1$.

\section{RESULTS}

A summary of the steps taken to numerically obtain the flowfield as the particle deposit increases are shown in a flowchart in Figure 6. The figure includes the 
relevant section numbers and equations. The deposit is build up in layers each of which has a non-dimensional width $\lambda$ at $\beta=0$.

\subsection{DIFFUSION REGIME}

In this section the situations when diffusion is the main mechanism of deposition are considered. In this case the particles are small and the parameter s is less than unity. Considering the physical conditions which satisfy $\mathrm{s}<1$ Figure 3 shows the parameter $\mathrm{s}$ as a function of particle size for various fibre sizes and freestream velocities. The region of interest in this section is that below the line $s=1$. It has been found in this work that for the conditions that satisfy $s<1$ the porosity of the deposit has a negligible effect upon the subsequent flow field and hence further deposition. An example of this is shown in Figure 7, where the streamlines of the flow are shown near a fibre that contains a layer of deposit of different porosities for conditions such that $s=0.875$. The streamlines obtained by assuming the deposit to be solid are also shown in the figures. In Figure 7a the porosity of the deposit, $\varphi$, is 0.9, i.e. $90 \%$ of the deposit is occupied by void space, and in Figure $7 \mathrm{~b} \varphi=0.95$.

As can be seen in Figure 7, the effect on the fluid motion due to the porosity of the deposit is very small for deposit made up of particles in the diffusion regime. This can be seen even when the porosity is $95 \%$ as shown in Figure $7 \mathrm{~b}$.

This can also be seen in Figure 8 where deposit build up on the filter is shown for the same conditions. This has been modelled using the steps outlined in Figure 6. In Figure 8 the deposit build up is shown for two layers assuming the porosity of the initial layer to be given by $\varphi=0.9$ and 0.95 and also assuming the deposit to be solid. As can be seen the effect of the deposit porosity is negligible particularly at the front of the fibre. Further layers of deposit have been determined using the numerical model and the same negligible effects of the porosity seen.

As the solution of the flow field for a fibre containing porous deposit is significantly more complex than for a solid deposit, it is computationally advantageous to assume that the deposit is solid. As the results show here, such an assumption is reasonable when considering particles in the diffusion regime, i.e. when the parameter $s$ is less than 1.

\subsection{INTERCEPTION REGIME}


Considering situations for which the parameter $s>1$, in this case the particles are larger than for the diffusion case and the main mechanism of deposition is interception. Also in these situations the permeability of the deposit is higher than for the diffusion case, as $\mathrm{k}$ is proportional to $\mathrm{d}_{\mathrm{p}}{ }^{2}$, equation (19), and more flow is seen to pass through it. This is seen in Figure 9 where the streamlines of the flow are shown near a fibre with a layer of deposit when $s=2.374, \varphi=0.95$. The streamlines obtained assuming that the layer is solid are also shown. As can be seen in this case the layer formed by the deposit only covers the front part of the fibre and the porosity is having a significant effect upon the flow. This can also be seen in Figure 10 where the growth of deposit is shown for the same case considered in Figure 9 for different deposit porosities. The effect of the porosity is to reduce the size of the region of the front of the fibre covered by deposit. It can be seen that the shape of the deposit predicted by the numerical model for all porosities is similar to that shown by Kanaoka et al (1986) (Figure 1) for the situation when inertial impaction is the main mechanism for deposition and not interception, the case considered here.

The case of pure interception in Figure 1 is at $\mathrm{Stk}=0, \mathrm{Pe}=\infty$ where the deposit width has a minimum at the front of the fibre. The numerical model developed here is not predicting this behaviour. An investigation into the modelled flow, and hence particle, behaviour in the vicinity of the fibre has been made. Streamlines of the flow given by values of the streamfunction with a constant difference $d \psi,\left(\psi_{\mathrm{i}}=\mathrm{id} \psi\right)$ so that an equivalent volume of flow is moving between the lines, have been considered. Assuming monodisperse particles with constant concentration the number of particles carried between any two streamlines $\psi_{\mathrm{i}}$ and $\psi_{\mathrm{i}+1}$ will be the same. Hence the width of the deposit layer will be proportional to the distance $d s_{i}$, where $d_{i}$ is the distance along the surface between the points where the particles travelling along the streamlines $\psi_{\mathrm{i}}$ and $\psi_{\mathrm{i}+1}$ impact the surface, see Figure 11a. It has been found in this work that for the particles in the interception region, $s>1$, the numerical model predicts that $\mathrm{ds}_{\mathrm{i}}$ increases with distance from the front of the fibre, i.e. with increasing $\psi$. This is seen in Figure $11 \mathrm{~b}$ where $\mathrm{ds}_{\mathrm{i}}$ is plotted as a function of $\psi_{\mathrm{i}}$ for $\mathrm{s}=2.374$, results have been shown for a clean fibre and also when a layer of deposit has already collected. As can be seen in both cases ds $s_{i}$ stays more or less constant until $\psi_{\mathrm{i}}$ gets close to the maximum value at which particles impact and then $\mathrm{ds}_{\mathrm{i}}$ increases rapidly. This corresponds to the deposit layer following the fibre shape until near the top 
where its width reduces, as seen in Figure 10. Once the initial deposit forms this shape on the front of the fibre, further deposition is found to follow the same pattern.

Various situations corresponding to $s>1$ have been considered, with various values for the deposits porosity, and the same behaviour is found to be predicted. This is different to the deposition patterns predicted by Kanaoka et al (1986) based on experimental work where it was found that the main areas of deposition were not around the front stagnation point but on the front face close to the $45^{\circ}$ point. A possible cause for this difference is the assumptions made in our model about the flow in the porous deposit. We have assumed that it can be modelled by Darcy's law and hence it is not possible to model the thin boundary layer in the porous deposit at the interface with the free flow. As the deposit starts to grow on the fibre the effects of this boundary layer may be significant. In order to investigate whether this could be the problem with the numerical model we have considered the deposit growth once a layer has already formed. The initial deposit has been assumed to form a shape similar to that shown by Kanaoka et al (1986), Figure 1 in the region $\mathrm{Stk}=0, \mathrm{Pe}=\infty$, with a flat surface facing the flow. An example of the flow around, and through such a layer is shown in Figure 12 for two different porosities, $\varphi=0.9,0.95$. Two streamlines for both porosities are shown for the case when $\kappa=0.05$, which corresponds to the parameters taken in the flow shown in Figure 9. Comparing the streamlines shown in Figures 12 and 9 it can be seen that the more streamlined shape formed by the deposit in Figure 9 enables the flow to adapt and hence less passes through the deposit. A plot of the length $\mathrm{ds}_{\mathrm{i}}$ as a function of $\psi_{\mathrm{I}}$ is shown in figure 13 for the case when an initial layer of deposit is assumed in the model (Figure 12) and also when the deposit is build up from a clean fibre (Figure 9). As can be seen when an initial layer of deposit is assumed then $\mathrm{ds}_{\mathrm{i}}$ initially decreases to a minimum and then increases again near the top range of $\psi_{\mathrm{i}}$. This results in deposit collecting on this layer in the shape shown in Figure 14. This is closer to the deposit growth identified by Kanaoka et al (1986) and demonstrates the sensitivity of the numerical model to the initial shape of the porous surface.

\section{CONCLUSIONS}

In this paper the numerical model developed earlier by the authors has been used to investigate the loading behaviour of filters for conditions such that diffusion, and also, 
interception, are the main deposition mechanisms. In the case of the smallest particles when diffusion is the dominant mechanism the model has shown to predict the pattern of deposition as that shown by Kanaoka et al (1986). Also the numerical model has shown that, in this case, the porosity of the deposit has an insignificant effect upon subsequent deposition. Hence, when modelling such situations it is reasonable to assume that the deposit collected forms a solid boundary to the flow, thus simplifying the model.

For larger particles when interception dominates as a mechanism of deposition, the model has shown that the porosity of the deposit has a more significant effect upon the flow and particle motion. However is has been found that the numerical model is not predicting the deposition patterns found previously by Kanaoka et al (1986) if the deposit is build up from a clean fibre. If however an initial deposit is assumed that has the same form as that found in the earlier work then subsequent build up predicted by the numerical model is found to follow the same pattern as found by others. It appears therefore that accurate description of the initial deposit is crucial in obtaining the correct morphology of the particulate deposit.

There are several possible reasons why the numerical model developed is not accurately modelling the flow in the initial stages of loading for particles in the interception region. As mentioned, one reason for this may be the assumptions made in the model. The situation modelled consists of two distinct flow regimes, porous and free flow, with the interface of the two regions occurring at the surface where particle deposition takes place. The modelling of such coupled free/porous flows is an important topic in engineering and has been the subject of many studies. The two distinct flow regimes are modelled by differential equations and the main problem is an accurate description of the flow at the interface. The flow in the porous region is governed by Darcy law, equation (6) and the flow in the free flow by Stokes flow, equation (2). There is a thin layer, of thickness of the order $\mathrm{k}^{1 / 2}$, within the porous media where the flow velocity evolves from its Darcy value to the interface value, similar to a boundary layer. The Darcy equation is not compatible with such a region as it has no shear stress associated with it. The approach adopted here to deal with this has been to adopt a modification of the Beavers and Joseph condition, equation (10). This introduces the slip coefficient $\alpha_{\mathrm{BJ}}$ which is dependent upon the structure of the porous region. As the thickness of this boundary layer is small its effect upon the flow 
field is generally insignificant. However in the initial stages of loading, when the deposit collected is small so that the size of the porous layer is small, the effect of this boundary layer may need to be considered and modelled more accurately. From

Figure 4 it can be seen that $k^{1 / 2}>d_{p}$ only for $\varphi>0.9$ and hence the thickness of the boundary layer is of the same size, or greater, than the size of the particles for these large porosities.

An alternative model of flow in the porous medium is provided by the Brinkman equation which takes into account this boundary layer. However it has been shown that the Brinkman equation accurately describes the flow only for high porosity media, $\varphi>0.95$, see Durlofsky and Brady (1987).

There are several other possibilities leading to a change of shape in the initial deposit. The porosity of the deposit has been shown to vary with depth, Rodríguez-Pérez et al (2005), and also with deposition mechanism, which leads to a dependence on angle. A second possibility is that slight deviations of particle trajectories from the flow, which can arise when Stk is small, will mainly affect trajectories near the front of the fibre and lead to more deposition away from $\beta=0$, see figure $2 b$.

Also the model assumes that a smooth porous layer is formed and hence the structure of the dendrites is not modelled. This is a possible source of error in the results. A final possible inaccuracy is the assumption of 2D fluid and particle motion. The work of Lehmann and Kasper (2002) demonstrated the importance of 3D simulations in the inertial regime region to obtain realistic particulate deposits. It is also possible that this may be the case in the interception region. All these possibilities are areas of possible further investigation.

\section{REFERENCES.}

Beavers, G.S. and Joseph, D.D. (1967) Boundary conditions at a naturally permeable wall. Journal of Fluid Mechanics. 30, 197-207.

Biggs, M.J., Humby, S.J., Buts, A. and Tuzun, U. (2003) Explicit numerical simulation of suspension flow with deposition in porous media: influence of local flow field variation on deposition processes predicted by trajectory methods. Chemical Engineering Science, 58, 1271-1288.

Brown, R.C. (1993) Air filtration. An integrated approach to the theory and application of fibrous filters, Pergamon Press, Oxford. 
Dunnett, S.J. and Clement, C.F. (2006) A numerical study of the effects of loading from diffusive deposition on the efficiency of fibrous filters. Journal of Aerosol Science 37, 1116-1139.

Dunnett, S.J. and Clement, C.F. (2009), A numerical model of fibrous filters containing deposit, Engineering analysis with boundary elements, 33 (5), 601-610 Durlofsky, L. and Brady, J.F. (1987) Analysis of the Brinkman equation as a model for flow in porous media, Physics of Fluids, 30, 3329-3341.

Filippova, O. and Hanel, D. (1997) Lattice-Boltzmann simulation of gas-particle flow in filters, Computers and Fluids, 26(7), 697-712.

Happel, J. (1959) Viscous flow relative to arrays of cylinders, American Institute of Chemical Engineers Journal, 5(2), 174-177

Hildyard, M.L., Ingham, D.B., Heggs, P.J. and Kelmanson, M.A. (1985) Integral equation solution of viscous flow through a fibrous filter, In Boundary Elements VII (ed. C.A.Brebbia and G.Maier), Springer-Verlag, Berlin.

Jung, Y. and Tien, C. (1993) Simulation of aerosol deposition in granular media. Aerosol Science and Technology, 18, 418-440.

Kanaoka,C., Emi, H., Hiragi,S., and Myojo, T. (1986) Morphology of particulate agglomerates on a cylindrical fiber and collection efficiency of a dust loaded fiber. In: Aerosols -Formation and Reactivity ( Proceedings $2^{\text {nd }}$ International Aerosol Conference, Berlin 1986 ).Pergamon Journals Ltd. Oxford, 674-677. Karadimos, A. and Ocone, R. (2003) The effect of the flow field recalculation on fibrous filter loading: a numerical simulation. Powder technology, 137, 109-119. Kasper, G., Schollmeier, S., Meyer, J. and Hoferer, J. (2009) The collection efficiency of a particle-loaded single filter fiber, Journal of Aerosol Science, 40, 993-1009 Kasper, G., Schollmeier, S., Meyer, J. (2010) Structure and density of deposits formed on filter fibers by inertial particle deposition and bounce, Journal of Aerosol Science $41,1167-1182$

Kim, S. and Russel, W.B. (1985) Modelling of porous media by renormalization of the Stokes equations, Journal of Fluid Mechanics, 154, 269-286. 
Kostoglou, M. and Konstandopoulos, A.G. (2000) Particulate deposit shape evolution on cylinders in cross-flow at high Stokes numbers, Journal of Aerosol Science, 31, pp427-436.

Kuwabara, S. (1959) The forces experienced by randomly distributed parallel circular cylinders or spheres in a viscous flow at small Reynolds numbers, Journal of the Physical Society of Japan, 14(4), 527-532.

Lehmann, M.J. and Kasper, G. (2002) CFD simulations of single fibre loading, In M.J. Lehmann, \& G. Kasper (Eds.), Particle Loading and Kinetics of Filtration in Fibrous Filters (pp. 91-98).Institutfur Mechanische Verfahrenstechnik und Mechanik Universitat Karlsruhe,2002ISBN:3-9805-220-2-4.

Li, S.-Q and Marshall, J.S. (2007) Discrete element simulation of micro-particle deposition on a cylindrical fiber in an array, Journal of Aerosol Science, 38, 10311046.

Payatakes, A.C. (1977) Model of transient aerosol particle deposition in fibrous media with dendritic pattern, American Chemical Engineering Journal, 23, pp192-202. Payatakes, A.C. and Gradon, L. (1980) Dendritic deposition of aerosols by convective Brownian motion for small, intermediate and high Knudsen numbers, American Chemical Engineering Journal, 26, pp443-454.

Przekop, R., Moskal, M., and Gradon, L. (2003) Lattice-Boltzmann approach for description of the structure of deposited particulate matter in fibrous filters. Journal of Aerosol Science, 34, 133-147.

Qian,F., Zhang, J. and Huang, Z. (2009) Effects of the operating conditions and geometry parameter on the filtration performance of a fibrous filter, Chemical Engineering Technology, 32(5), 789-797.

Rodríguez-Pérez, D., Castillo, J.I. and Antoranz, J.C. (2005) Relationship between particle deposit characteristics and the mechanism of particle arrival, Physics Review E 72021403 1-9.

Schweers,E. and Löffler (1994) Realistic modelling of the behaviour of fibrous filters through consideration of filter structure. Powder Technology 80, 191-206.

Stechkina, I.B. and Fuchs, N.A. (1966) Studies on aerosol fibrous filters-I.

Calculations of diffusive deposition of aerosols in fibrous filters. Annals of Occupational Hygiene 9, 59-64.

Vainshtein, P., Shapiro, M., Gutfinger, C. (2004) Mobility of permeable aggregates: effects of shape and porosity. Aerosol Science and Technology 35, 383-404. 
Vanni, M. (2000) Creeping flow over spherical permeable aggregates, Chemical Engineering Science, 55, 685-698. 